\title{
Local master equations may fail to describe dissipative critical behavior
}

\author{
Michael Konopik and Eric Lutz $\odot$ \\ Institute for Theoretical Physics I, University of Stuttgart, D-70550 Stuttgart, Germany
}

(Received 12 January 2021; accepted 9 February 2022; published 2 March 2022)

\begin{abstract}
Local quantum master equations provide a simple description of interacting subsystems coupled to different reservoirs. They have been widely used to study nonequilibrium critical phenomena in open quantum systems. We here investigate the validity of such a local approach by analyzing a paradigmatic system made of two harmonic oscillators each in contact with a heat bath. We evaluate the steady-state mean occupation number for varying temperature differences and find that local master equations generally fail to reproduce the results of an exact quantum-Langevin-equation description. We relate this property to the inability of the local scheme to properly characterize intersystem correlations, which we quantify with the quantum mutual information.
\end{abstract}

DOI: 10.1103/PhysRevResearch.4.013171

\section{INTRODUCTION}

Quantum master equations have been instrumental in the study of open quantum systems since their introduction by Pauli in 1928 [1]. They offer powerful, yet approximate, means to describe the time evolution of the reduced density operator of quantum systems coupled to external environments [2-5]. They allow the analysis of the dynamics of both diagonal density matrix elements (populations), involved in thermalization processes and of nondiagonal densitymatrix elements (coherences), associated with dephasing phenomena. As a consequence, they have found widespread application in many different areas, ranging from quantum optics [6] and condensed-matter physics [7] to nonequilibrium statistical mechanics [8] and quantum information theory [9].

In the past decade, quantum master equations have become a popular tool to investigate nonequilibrium phase transitions that occur between (detailed-balance breaking) steady states [10-28]. Special attention has been given to two broad classes of out-of-equilibrium phase transitions: (i) those induced by external driving fields in systems interacting with a single bath (driven-dissipative processes) [10-16] and (ii) those generated by the coupling of a system to several baths (boundary-driven processes) [17-28]. Remarkably, nontrivial exact analytical steady-state solutions of local quantum Lindblad master equations have been obtained for various many-body spin-chain models [17,18,21-24,26,28], thus, offering new insight into boundary-driven critical systems.

However, the form of the quantum master equations employed in these studies is often postulated. Their validity is, thus, not completely clear a priori. This is especially true

Published by the American Physical Society under the terms of the Creative Commons Attribution 4.0 International license. Further distribution of this work must maintain attribution to the author(s) and the published article's title, journal citation, and DOI. for boundary-driven processes where the system of interest is coupled to several reservoirs. In this case, it has recently been shown that local master equations, that are commonly used to examine nonequilibrium phase transitions [17-28], may violate the second law of thermodynamics [29] and give rise to nonphysical results, such as incorrect steady-state distributions or nonzero currents for vanishing bath interactions [30-40], even in the limit of small bath couplings. These inconsistencies are related to the fact that local quantum master equations, whose total dissipator is simply the sum of the single-bath dissipators, incorrectly neglect bath-bath correlations, which are induced by intersystem interactions, in contrast to global quantum master equations [29-40]. Interestingly, the local approach has been shown to provide a better description of quantum heat engines than the global approach in some parameter regimes [35]. Meanwhile, the validity of Lindblad quantum master equations has, for example, been discussed in the context of quantum transport [41,42], quantum relaxation $[43,44]$, and entanglement generation [45]. But these results cannot be straightforwardly extended to nonequilibrium phase transitions as the considered models do not exhibit critical behavior.

In this paper, we examine the accuracy of a quantummaster-equation description of dissipative critical phenomena by analyzing an exemplary system consisting of two interacting harmonic oscillators, each weakly coupled to a thermal reservoir. This system naturally appears in many areas, most notably in cavity optomechanics [46]. Manybody superradiant phase-transition models, such as the Dicke model [47] and the Tavis-Cummings model [48], can also be mapped onto such a system after a Holstein-Primakoff transformation [49,50]. We concretely compare local and global quantum master equations with and without rotating-wave approximation for the oscillator-oscillator interaction to exact results provided by a quantum-Langevin-equation description [42-45]. We explicitly evaluate the stationary mean occupation number of one oscillator for various nonequilibrium temperature differences. We find that the local master equation generally fails to reproduce the results of the quantum 
Langevin equation especially for large temperature differences, whereas the global approach exhibits better agreement. We show that this feature is directly related to the inability of the local description to correctly capture intersystem correlations, which we quantify with the help of the quantum mutual information [9].

\section{COUPLED-OSCILLATOR MODEL}

We consider a system of two interacting harmonic oscillators with a Hamilton operator,

$$
H=\sum_{j=1,2} \omega_{j} a_{j}^{\dagger} a_{j}+\lambda\left(a_{1} a_{2}^{\dagger}+a_{1}^{\dagger} a_{2}\right)+\kappa\left(a_{1} a_{2}+a_{1}^{\dagger} a_{2}^{\dagger}\right),
$$

where $a_{j}^{\dagger}$ and $a_{j}$ are the usual ladder operators and $\omega_{j}$ is the respective frequencies. We will examine two different types of intersystem interactions: (i) a position-position interaction, $x_{1} x_{2}$, corresponding to $\kappa=\lambda$, and (ii) its rotating-wave version, obtained for $\kappa=0$. Two important points should be stressed: First, the position-position coupling $x_{1} x_{2}$ leads to critical behavior above a critical interaction strength [51-53], in contrast to the commonly treated Hookian interaction $\left(x_{1}-\right.$ $\left.x_{2}\right)^{2}$ [41-45]. In addition, although the rotation-wave approximation is usually associated with a weak-coupling condition, $\lambda / \omega_{i} \ll 1$, it has recently been shown that counter-rotating terms may be effectively suppressed in modulated systems, even in the ultrastrong regime [54,55]. This opens the possibility to experimentally study critical behavior in strongly interacting rotating-wave models.

The isolated Hamilton operator (1) may be diagonalized exactly for both intersystem interactions, yielding two uncoupled modes with respective energies [29,51,52],

$$
\begin{gathered}
\omega_{ \pm}^{\mathrm{pp}}=\left\{\left[\omega_{1}^{2}+\omega_{1}^{2} \pm \sqrt{\left(\omega_{1}^{2}-\omega_{2}^{2}\right)^{2}+16 \lambda^{2} \omega_{1} \omega_{2}}\right] / 2\right\}^{1 / 2}, \\
\omega_{ \pm}^{\mathrm{rw}}=\left[\omega_{1}+\omega_{1} \pm \sqrt{\left(\omega_{1}-\omega_{2}\right)^{2}+4 \lambda^{2}}\right] / 2
\end{gathered}
$$

These energies display critical behavior at the respective critical couplings $\lambda_{\mathrm{c}}^{\mathrm{pp}}=\sqrt{\omega_{1} \omega_{2}} / 2$ and $\lambda_{\mathrm{c}}^{\mathrm{rw}}=\sqrt{\omega_{1} \omega_{2}}$. Above these points, the eigenfrequencies of the Hamilton operator (1) become imaginary or negative. The energy spectrum is, thus, no longer bounded from below. These critical values are in agreement with those of the Dicke and Tavis-Cummings models [56-58].

We next attach each quantum harmonic oscillator to a heat bath with respective temperature $T_{j}$ (Fig. 1). As commonly performed we model these reservoirs by an ensemble of harmonic oscillators [2-5]. We further assume that the system-bath coupling is weak so that the rotating-wave approximation is applicable to that coupling (see Appendix A). We reemphasize that the interaction between the two harmonic oscillators of the system (1) might be strong.

Most studies of dissipative phase transitions consider complex interacting many-body systems [17-28]. The direct comparison between global and local master equation descriptions is, thus, extremely difficult in these systems. By contrast, the coupled-oscillator model is complicated enough to exhibit steady-state critical behavior and, at the same time, simple enough to allow for: (i) a detailed comparison between global

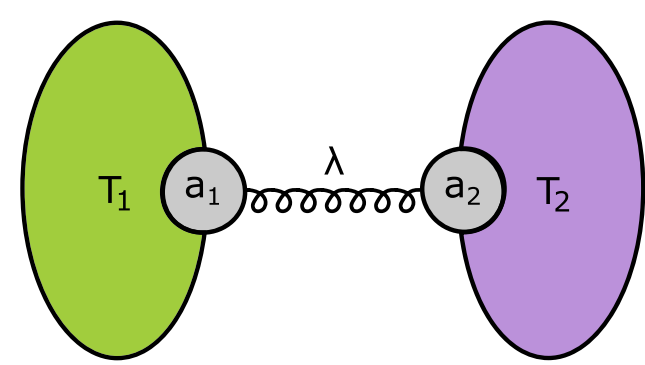

FIG. 1. Coupled-oscillator model. Two quantum harmonic oscillators interact with each other with interaction strength $\lambda$. Each of them is weakly coupled with a heat bath with respective temperature $T_{j}(j=1,2)$. A nonequilibrium steady state is established when the two temperatures are different, and heat flows from one oscillator to the other.

and local approaches and (ii) the evaluation of intersystem correlations.

\section{QUANTUM-MASTER-EQUATION DESCRIPTION}

In the usual Born-Markov limit, the density operator $\rho$ of the joint quantum system obeys a Lindblad master equation of the form [2-5] (we set $\hbar=1$ throughout),

$$
\dot{\rho}=-i[H, \rho]+\sum_{k=1,2} \sum_{i=1-4} \sum_{j=1-4} \mathcal{D}_{k}\left(A_{i}, A_{j}\right),
$$

where the dissipators are given by $\mathcal{D}_{k}\left(A_{i}, A_{j}\right)=$ $\Gamma_{k}\left(A_{i}, A_{j}\right)\left(A_{i} \rho A_{j}-\left\{A_{j} A_{i}, \rho\right\} / 2\right)$. The coefficients $\Gamma_{k}\left(A_{i}, A_{j}\right)$ as well as the operators $A_{i}$ depend on the local or global type of the quantum master equation [29].

In the local approach, each oscillator interacts with its heat bath (labeled by $k=1$ or 2) as if it were not coupled to the other oscillator. As a result, the quantum master equation may be derived as usual in the local eigenbasis of one oscillator [2-5]. The operators $A_{i}$ are here the standard ladder operators, $\left(a_{1}, a_{1}^{\dagger}, a_{2}, a_{2}^{\dagger}\right)$, and the dissipators are given by $\Gamma_{k}\left(a_{i}, a_{j}^{\dagger}\right)=$ $\delta_{k j} \delta_{i j} \gamma_{k}\left[N\left(\omega_{j}, \beta_{k}\right)+1\right]$ and $\Gamma_{k}\left(a_{i}^{\dagger}, a_{j}\right)=\delta_{k j} \delta_{i j} \gamma_{k} N\left(\omega_{j}, \beta_{k}\right)$, where $\gamma_{k}$ is the damping coefficient of bath $k$ and $N\left(\omega_{j}, \beta_{k}\right)=$ $1 /\left[\exp \left(\beta_{k} \omega_{j}\right)-1\right]$ denotes the thermal occupation number [2-5]. These formulas evidently hold for the two kinds of oscillator-oscillator interaction in Eq. (1).

On the other hand, the global master equation is derived in the global eigenbasis of the combined two-oscillator system $[29,43]$. The diagonalization of the joint Hamilton operator (1) accounts for the indirect subsystem-reservoir and reservoirreservoir correlations which are generated by their coupling to the system. Such correlations are ignored in the local approach. This is the reason why the local master equation may violate the second law of thermodynamics [29]. The explicit (and lengthy) expressions for the dissipators are summarized for both the position-position and rotating-wave interactions in Appendix A. In this situation, they depend on operators $A_{i}$ that are given by properly rotated ladder operators.

In the following, we will solve the four different quantum master equations (global/local forms with/without rotatingwave interaction) by applying a characteristic function method in symplectic space (see Appendix A) and evaluate the 
steady-state mean occupation number $\left\langle a_{j}^{\dagger} a_{j}\right\rangle_{\mathrm{ss}}=\operatorname{tr}\left(\rho_{\mathrm{ss}} a_{j}^{\dagger} a_{j}\right)$, where $\rho_{\mathrm{ss}}$ is the stationary density operator.

\section{QUANTUM-LANGEVIN-EQUATION DESCRIPTION}

In order to assess its validity both for equilibrium and nonequilibrium conditions, we will compare the steady-state properties of the approximate quantum-master-equation treatment to those of the exact quantum-Langevin-equation approach [3]. To this end, we will extend the results obtained for Hookian coupling [42-45] to the position-position and rotating-wave interactions of Eq. (1). The quantum Langevin equations read [3]

$$
\dot{a}_{j}=-i\left[a_{j}, H\right]-\gamma_{j} a_{j}+\sqrt{2 \gamma_{j}} a_{j, \text { in }},
$$

where the noisy input operators $a_{j, \text { in }}$, stemming from the interaction with the respective baths, are characterized by the correlation function in Fourier space,

$$
\begin{gathered}
\left\langle a_{j, \text { in }}\left(v_{j}\right) a_{j, \text { in }}^{\dagger}\left(v_{j}^{\prime}\right)\right\rangle=2 \gamma_{j}\left[N\left(v_{j}, \beta_{j}\right)+1\right] \delta\left(v_{j}-v_{j}^{\prime}\right), \\
\left\langle a_{j, \text { in }}^{\dagger}\left(v_{j}^{\prime}\right) a_{j, \text { in }}\left(v_{j}\right)\right\rangle=2 \gamma_{j} N\left(v_{j}, \beta_{j}\right) \delta\left(v_{j}-v_{j}^{\prime}\right) .
\end{gathered}
$$

The coupled quantum Langevin equations (5) can be solved by matrix inversion in Fourier space (see Appendix C). In particular, the mean occupation number is here equal to

$$
\left\langle a_{j}^{\dagger} a_{j}\right\rangle_{\text {Langevin }}=\int_{-\infty}^{\infty} \int_{-\infty}^{\infty}\left\langle a_{j}^{\dagger}\left(v_{j}\right) a_{j}\left(v_{j}^{\prime}\right)\right\rangle e^{i\left(v_{j}-v_{j}^{\prime}\right) t} d v_{j} d v_{j}^{\prime} .
$$

Equation (8) is independent of time in the steady-state regime, and we will set $t=0$ in the following. Steady-state mean occupation numbers may be evaluated exactly (without any approximations) in the quantum-Langevin-equation formalism in contrast to the quantum-master-equation approach [42].

\section{RESULTS}

Figure 2 presents the steady-state mean occupation number $\left\langle a_{1}^{\dagger} a_{1}\right\rangle_{\text {ss }}$ of the first oscillator as a function of the reduced interaction strength $\lambda / \lambda_{\mathrm{c}}$ in the equilibrium (high-temperature) case $\Delta T=T_{2}-T_{1}=0$. We observe perfect agreement between the global quantum master equation (blue dots), the quantum Langevin equation (green line), and the equilibrium (Gibbs) state $\rho_{\mathrm{eq}}=\exp (-\beta H) / Z$ (yellow line) for all values of $\lambda / \lambda_{\mathrm{c}}$ for both the position-position and the rotating-wave (inset) interactions. By contrast, the local quantum master equation (orange squares) deviates from these results as the critical point is approached; noticeably, it does not exhibit any critical behavior at all for the intersystem rotating-wave interaction (the inset).

In order to gain deeper insight on the nonequilibrium properties of the different quantum master equations, we next examine the ratio of their steady-state mean occupation numbers and the corresponding quantum-Langevin-equation expressions $\left\langle a_{1}^{\dagger} a_{1}\right\rangle_{\mathrm{ss}} /\left\langle a_{1}^{\dagger} a_{1}\right\rangle_{\text {Langevin }}$ for increasing temperature differences $\Delta T$. In the high-temperature regime $\left(\beta_{i} \omega_{i} \ll 1\right)$, Fig. 3(a) shows that, for the position-position interaction, the local approach gets worse as the system moves further away from equilibrium and that even the global approach slightly

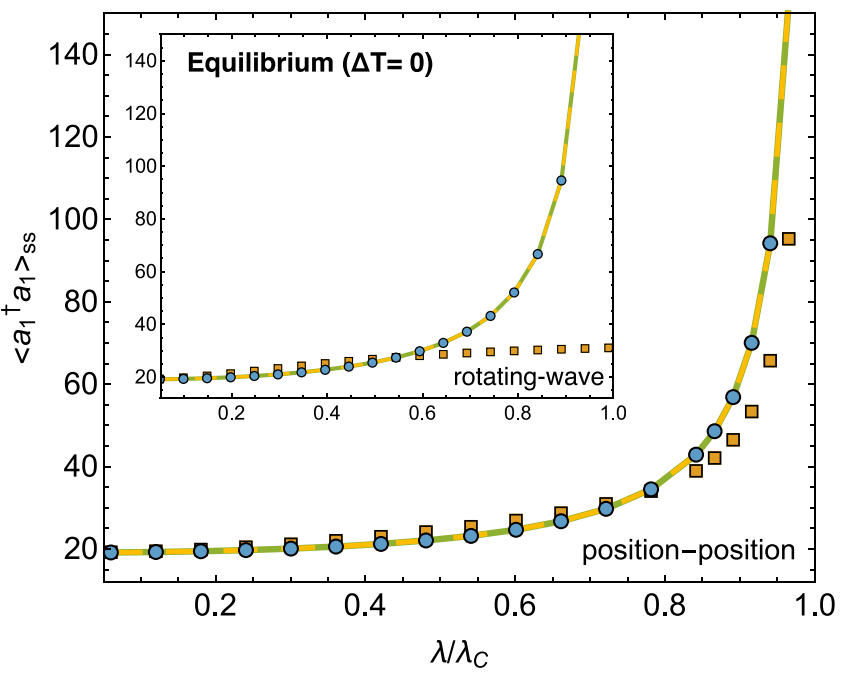

FIG. 2. Steady-state mean occupation number $\left\langle a_{1}^{\dagger} a_{1}\right\rangle_{\mathrm{ss}}$ of the first oscillator as a function of the dimensionless interoscillator interaction strength $\lambda / \lambda_{\mathrm{c}}$ for the equilibrium (high-temperature) case $\Delta T=$ 0 . For the position-position interaction [see Eq. (1)], the results of the global quantum master equation (blue dots) perfectly agree with those of the quantum Langevin equation (green line) as well as those of the Gibbs state $\rho_{\mathrm{eq}}=\exp (-\beta H) / Z$ (yellow line), whereas those of the local quantum master equation deviate more and more as the critical point is approached. For the rotating-wave interaction (the inset), the global approach still perfectly matches the predictions of the quantum Langevin equation, whereas the local scheme does not display any critical behavior. Parameters are $\gamma_{1}=\gamma_{2}=1.5 \times 10^{-4}$, $\omega_{1}=5, \omega_{2}=2$, and $T_{1}=T_{2}=98$.

departs from the predictions of the quantum Langevin equation for large $\Delta T$. A similar behavior is seen in Fig. 3(b) when the two temperatures are low $\left(\beta_{i} \omega_{i} \gg 1\right)$. Analogous results are displayed for the rotating-wave interaction in Figs. 4(a) and 4(b): Remarkably, the global quantum master equation here always perfectly matches the quantum Langevin equation for all $\lambda$ and all $\Delta T$, whereas the local quantum master equation always fails to describe critical behavior. We also mention that the discrepancy between the various descriptions, in general, depends on the sign of the nonequilibrium temperature difference $\Delta T$ (see Appendix D).

The success/failure of the quantum-master-equation description of dissipative critical phenomena may be understood both physically and mathematically. To first address the physical aspect, we consider the quantum mutual information between the two harmonic oscillators $I(\rho)=S\left(\rho_{1}\right)+$ $S\left(\rho_{2}\right)-S(\rho)$, where $S\left(\rho_{i}\right)=-\operatorname{tr}\left\{\rho_{i} \ln \rho_{i}\right\}$ is the von Neumann entropy and $\rho_{i}=\operatorname{tr}_{i} \rho$ are the reduced density operators of the respective harmonic oscillators [9]. The quantum mutual information is a measure of the total (classical and quantum) correlations between two subsystems and has been used broadly to characterize critical transitions [59-63]. Figure 5 shows that the stationary quantum mutual information $I\left(\rho_{\mathrm{ss}}\right)$ displays a very similar dependence on the interaction strength $\lambda$ as the average occupation number $\left\langle a_{1}^{\dagger} a_{1}\right\rangle_{\mathrm{ss}}$ represented in Fig. 1 both for the position-position and rotating-wave interoscillator interactions (see Appendix B). The shortcomings of the quantum-master-equation approach, 


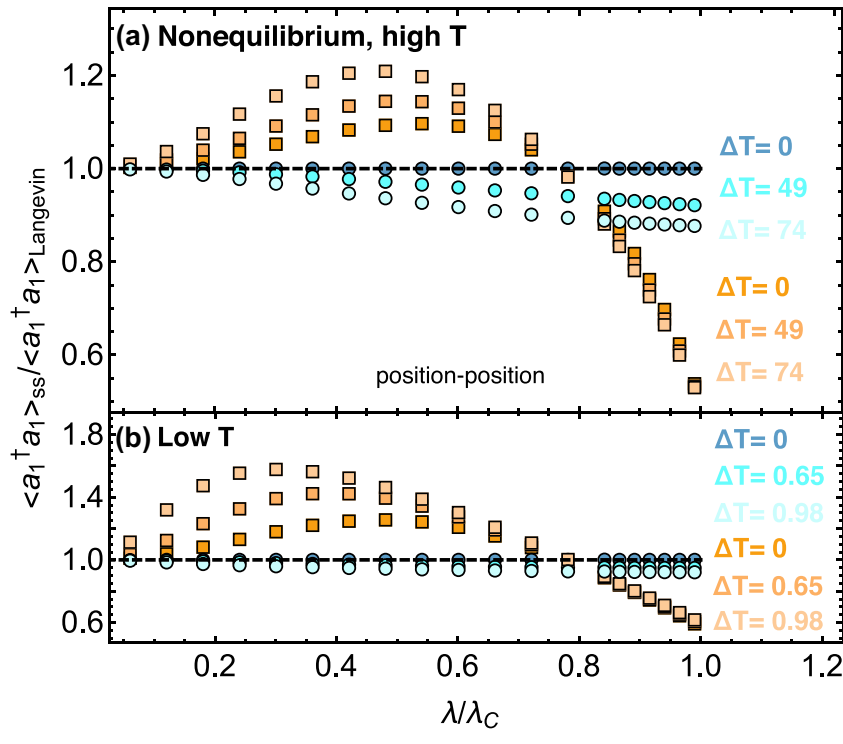

FIG. 3. Ratio of the steady-state mean occupation numbers $\left\langle a_{1}^{\dagger} a_{1}\right\rangle_{\mathrm{ss}} /\left\langle a_{1}^{\dagger} a_{1}\right\rangle_{\text {Langevin }}$ of the quantum master equation and the quantum Langevin equation as a function of $\lambda / \lambda_{c}$ for various nonequilibrium temperature differences $\Delta T$ and position-position interaction [see Eq. (1)]. (a) In the high-temperature regime $\left(\beta_{i} \omega_{i} \ll\right.$ 1 ), the local (global) quantum master equation [orange squares (blue dots)] strongly (slightly) departs from the predictions of the quantum Langevin equation for increasing temperature differences, missing the critical behavior for all $\Delta T$. (b) An analogous behavior is observed for low temperatures $\left(\beta_{i} \omega_{i} \gg 1\right)$. Same parameters as in Fig. 2.

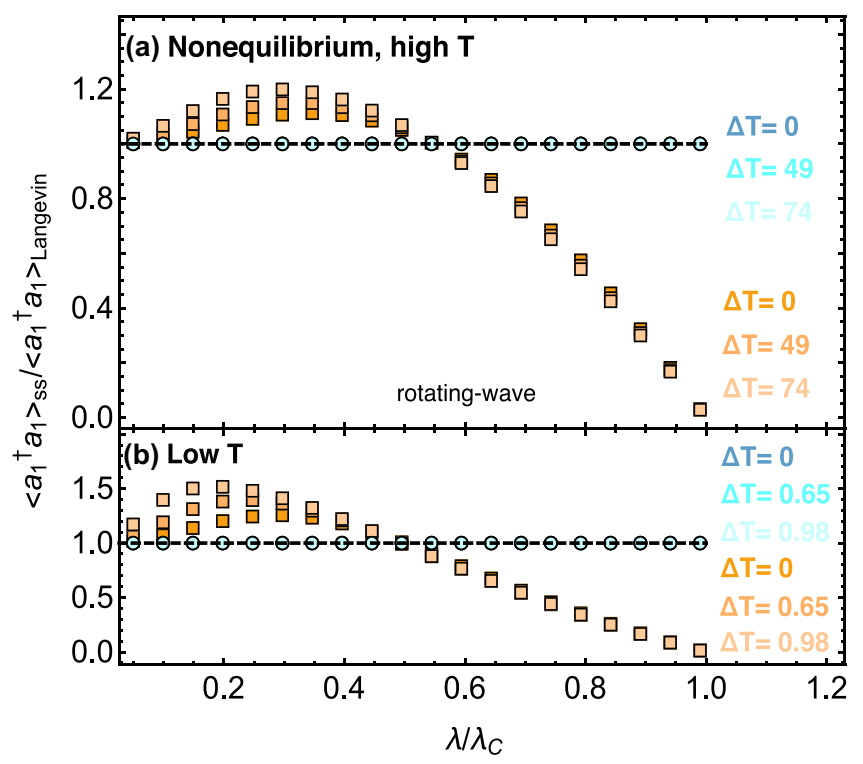

FIG. 4. Ratio of the steady-state mean occupation numbers $\left\langle a_{1}^{\dagger} a_{1}\right\rangle_{\mathrm{ss}} /\left\langle a_{1}^{\dagger} a_{1}\right\rangle_{\text {Langevin }}$ of the quantum master equation and the quantum Langevin equation as a function of $\lambda / \lambda_{\mathrm{c}}$ for various temperature differences $\Delta T$ and rotating-wave interaction [see Eq. (1)]. Both for (a) high temperatures $\left(\beta_{i} \omega_{i} \ll 1\right)$ and (b) low temperatures $\left(\beta_{i} \omega_{i} \gg\right.$ $1)$, the global (blue dots) quantum master equation exactly agrees with the quantum Langevin equation, whereas the local quantum master equation (orange squares) shows large deviations for increasing temperature differences. Same parameters as in Fig. 2, with $T_{2}=1.96$ and $T_{1}=T_{2}-\Delta T$ in the low-temperature limit.

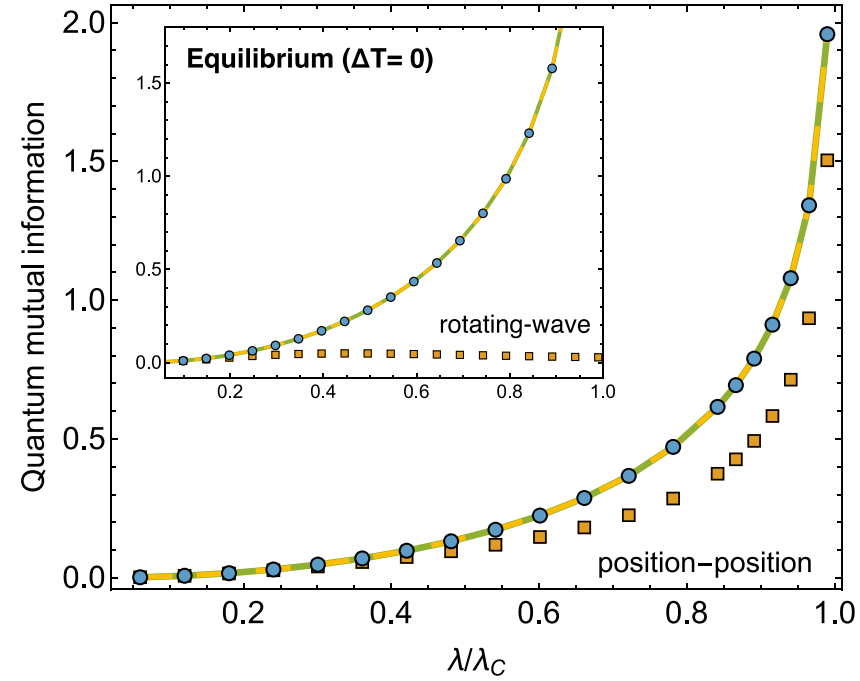

FIG. 5. Steady-state quantum mutual information $I\left(\rho_{\mathrm{ss}}\right)$ of the two-oscillator system as a function of the dimensionless interoscillator interaction strength $\lambda / \lambda_{\mathrm{c}}$ for the equilibrium (high-temperature) case $\Delta T=0$. The mutual information displays an analogous dependence of the interaction strength as the steady-state mean occupation number $\left\langle a_{1}^{\dagger} a_{1}\right\rangle_{\mathrm{ss}}$ shown in Fig. 1. Same parameters as in Fig. 2.

especially in its local version, may, thus, be traced to its inability to correctly capture intersystem correlations close to the critical point. This feature can be confirmed mathematically by looking at the way the respective Lindblad quantum master equations are obtained [29]: The dissipators in the local master equation are indeed derived in the local eigenbasis of each separate harmonic oscillator, whereas those of the global master equation follow from a diagonalization of the interacting two-oscillator system [the unitary evolution given by the von Neumann term in Eq. (4) describes coupled dynamics in both cases]. The global scheme, thus, better accounts for intersystem correlations than the local one and should, therefore, be preferred. Such intersystem correlations are indeed crucial for an accurate description of many-body critical systems and should not be incorrectly omitted. Yet, despite these deficiencies, local quantum master equations have been a tool of choice in numerous studies on dissipative critical behavior [17-28].

\section{CONCLUSIONS}

We have examined the ability of global and local quantum master equations to accurately describe dissipative critical phenomena using an illustrative system of two interacting damped harmonic oscillators with and without rotating-wave interaction. This model provides a transparent, yet generic, example to perform such a study. We have found that although the global master equation reproduces the results of the quantum Langevin equation reasonably well, the local version usually fails to do so, especially in the far-from-equilibrium regime; it generally fails in the case of the rotating-wave interaction. We have related these properties to the inability of the local approach to correctly apprehend oscillator-oscillator correlations that we have quantified with the help of the quantum 
mutual information. The latter quantity could be easily determined in the present two-oscillator model, in contrast to more complex interacting many-body systems. Our findings show that approximate local quantum master equations, in general, and their exact analytical solutions, in particular, should be used with caution when studying dissipative critical behavior and that the more complicated global approach should be favored instead.

\section{ACKNOWLEDGMENTS}

We acknowledge financial support from the German Science Foundation (DFG) (Contract No. FOR 2724).

\section{APPENDIX A: QUANTUM MASTER EQUATIONS}

In the following, we provide details about the dissipators of the four quantum master equations that we consider in our study (global/local forms with/without rotating-wave interaction) as well as their solutions.

The standard dissipators of the local quantum master equation are given below Eq. (4) in the main text. They are derived in the local eigenbasis of each oscillator [2-5] and, thus, hold for both the position-position and rotating-wave intersystem interactions. By contrast, the global master equations are derived in the global eigenbasis of the combined two-oscillator system obtained by diagonalizing the quadratic Hamilton operator $H$. The respective dissipators are then computed by expanding the system-bath interaction in this basis. We concretely consider the total system-bath Hamilton operator,

$$
H_{\text {tot }}=H+H_{S B 1}+H_{S B 2}+H_{B 1}+H_{B 2},
$$

with harmonic thermal baths $H_{B i}=\sum_{j} \omega_{i j} b_{i j}^{\dagger} b_{i j}$ and local system-bath couplings $H_{S B i}=\sum_{j} \kappa_{i j}\left(a_{i} b_{i j}^{\dagger}+\right.$ H.c. $)$ with coupling constants $\kappa_{i j}(i=1,2)$ [2-5].

In the case of the rotating-wave interaction, the diagonalization of $H$ leads to $H_{\mathrm{rw}}=\omega_{+}^{\mathrm{rw}} d_{+}^{\dagger} d_{+}+\omega_{-}^{\mathrm{rw}} d_{-}^{\dagger} d_{-}$with the eigenfrequencies $\omega_{ \pm}^{\mathrm{rw}}$ given in Eq. (3) of the main text and the rotated operators $d_{-}=a_{2} \cos \theta-a_{1} \sin \theta$ and $d_{+}=a_{1} \cos \theta+a_{2} \sin \theta$ where the angle $\theta$ satisfies $\cos ^{2} \theta=$ $\left(\omega_{+}^{\mathrm{rw}}-\omega_{-}^{\mathrm{rw}}\right) /\left(\omega_{+}^{\mathrm{rw}}-\omega_{-}^{\mathrm{rw}}\right)$ [29]. The global Lindblad dissipators then follow as [29]

$$
\begin{gathered}
\Gamma\left(a_{1}, a_{1}^{\dagger}\right)=\gamma_{1}^{+} c^{4}+\gamma_{1}^{-} s^{4}+\left(\gamma_{2}^{+}+\gamma_{2}^{-}\right) c^{2} s^{2} \\
\Gamma\left(a_{1}^{\dagger}, a_{1}\right)=\gamma_{1}^{+\prime} c^{4}+\gamma_{1}^{-\prime} s^{4}+\left(\gamma_{2}^{+\prime}+\gamma_{2}^{-\prime}\right) c^{2} s^{2} \\
\Gamma\left(a_{2}, a_{2}^{\dagger}\right)=\gamma_{2}^{-} c^{4}+\gamma_{2}^{+} s^{4}+\left(\gamma_{1}^{+}+\gamma_{1}^{-}\right) c^{2} s^{2} \\
\Gamma\left(a_{2}^{\dagger}, a_{2}\right)=\gamma_{2}^{+\prime} c^{4}+\gamma_{2}^{-\prime} s^{4}+\left(\gamma_{1}^{+\prime}+\gamma_{1}^{-\prime}\right) c^{2} s^{2} \\
\Gamma\left(a_{1}, a_{2}^{\dagger}\right)=\gamma_{1}^{+} c^{3} s-\gamma_{1}^{-} s^{3} c+\gamma_{2}^{+} c s^{3}-\gamma_{2}^{-} c^{3} s \\
\Gamma\left(a_{1}^{\dagger}, a_{2}\right)=\gamma_{1}^{+\prime} c^{3} s-\gamma_{1}^{-\prime} s^{3} c+\gamma_{2}^{+\prime} c s^{3}-\gamma_{2}^{-\prime} c^{3} s
\end{gathered}
$$

with $c=\cos \theta, s=\sin \theta, \gamma_{i}^{ \pm}=\gamma, \gamma_{i}^{ \pm \prime}=\gamma_{i}^{ \pm} e^{-\beta_{i} \omega_{ \pm}}$, and $\Gamma\left(a_{1}^{\dagger}, a_{2}\right)=\Gamma\left(a_{2}^{\dagger}, a_{1}\right), \Gamma\left(a_{1}, a_{2}^{\dagger}\right)=\Gamma\left(a_{2}, a_{1}^{\dagger}\right)$.

In the case of the position-position interaction, the diagonalization of $H$ is more involved as it couples all four ladder operators with each other $\left(a_{1}, a_{2}, a_{1}^{\dagger}, a_{2}^{\dagger}\right)=S\left(c_{1}, c_{2}, c_{1}^{\dagger}, c_{2}^{\dagger}\right)$ [51]. The $4 \times 4$ diagonalization matrix $S$ is partitioned into four blocks with the $2 \times 2$ matrix $A$ on the diagonal blocks and $2 \times 2$ matrix $B$ matrix on the off-diagonal blocks,

$$
\begin{aligned}
& A=\left(\begin{array}{cc}
\frac{\left(\omega_{+}^{\mathrm{pp}}+\omega_{1}\right) \cos \theta}{2 \sqrt{\omega_{+}^{\mathrm{pp}} \omega_{1}}} & \frac{-\left(\omega_{-}^{\mathrm{pp}}+\omega_{1}\right) \sin \theta}{2 \sqrt{\omega_{-}^{\mathrm{p}} \omega_{1}}} \\
\frac{\left(\omega_{+}^{\mathrm{pP}}+\omega_{2}\right) \sin \theta}{2 \sqrt{\omega_{+}^{\mathrm{pp}} \omega_{2}}} & \frac{-\left(\omega_{-}^{\mathrm{pP}}+\omega_{2}\right) \sin \theta}{2 \sqrt{\omega_{-}^{\mathrm{p}} \omega_{2}}}
\end{array}\right), \\
& B=\left(\begin{array}{cc}
\frac{\left(-\omega_{+}^{\mathrm{pp}}+\omega_{1}\right) \cos \theta}{2 \sqrt{\omega_{+}^{\mathrm{pp}} \omega_{1}}} & \frac{\left(\omega_{-}^{\mathrm{pp}}-\omega_{1}\right) \sin \theta}{2 \sqrt{\omega_{-}^{\mathrm{pp}} \omega_{1}}} \\
\frac{\left(-\omega_{+}^{\mathrm{pp}}+\omega_{2}\right) \sin \theta}{2 \sqrt{\omega_{+}^{\mathrm{pp}} \omega_{2}}} & \frac{\left(\omega_{-}^{\mathrm{pp}}-\omega_{2}\right) \sin \theta}{2 \sqrt{\omega_{-}^{\mathrm{pp}} \omega_{2}}}
\end{array}\right),
\end{aligned}
$$

with the eigenfrequencies $\omega_{ \pm}^{\mathrm{pp}}$ given in Eq. (2) of the main text. The global Lindblad dissipators are then $\Gamma\left(A_{i}, A_{j}\right)=\Gamma_{1,13}^{i j}+$ $\Gamma_{1,31}^{i j}+\Gamma_{2,24}^{i j}+\Gamma_{2,42}^{i j}$ with

$$
\begin{aligned}
\Gamma_{1, k l}^{i j}= & \gamma_{1} N\left(\omega_{+}, \beta_{1}\right) S_{1}^{k} S_{3}^{l} W_{i}^{1} W_{j}^{3} \\
& +\gamma_{1} N\left(\omega_{-}, \beta_{1}\right) S_{2}^{k} S_{4}^{l} W_{i}^{2} W_{j}^{4} \\
& +\gamma_{1}\left[N\left(\omega_{+}, \beta_{1}\right)+1\right] S_{1}^{k} S_{3}^{l} W_{i}^{3} W_{j}^{1} \\
& +\gamma_{1}\left[N\left(\omega_{-}, \beta_{1}\right)+1\right] S_{2}^{k} S_{4}^{l} W_{i}^{4} W_{j}^{2}
\end{aligned}
$$

for the quantum oscillator 1 coupled to bath 1 at inverse temperature $\beta_{1}$ with $W=S^{-1}$. Here the indices $i, j$ run over $1-4$, corresponding to the elements of $\left(a_{1}, a_{2}, a_{1}^{\dagger}, a_{2}^{\dagger}\right)$ and $k, l$ run over all combinations of 1,3 . The indices $k, l$ correspond to the initially chosen local coupling terms in the derivation of the master equation before the diagonalization is applied, which can be ordered either as $a_{i} a_{i}^{\dagger}$ or $a_{i}^{\dagger} a_{i}$. Thus, there are 32 different terms corresponding to the 16 unique operator orderings $A_{i} A_{j}$ in the dissipators. Expressions for second bath at inverse temperature $\beta_{2}$ are analogous with $k, l$ now combinations of 2,4 .

We explicitly solve the linear local and global quantum master equations by computing the first and second moments of $\rho$ in symplectic space [2]. The symmetric characteristic function is defined by $\chi\left(\alpha_{1}, \alpha_{2}\right)=\left\langle D_{1}\left(\alpha_{1}\right) \otimes D_{2}\left(\alpha_{2}\right)\right\rangle$, where $D_{i}\left(\alpha_{i}\right)=\exp \left(\alpha_{i} a_{i}^{\dagger}-\alpha_{i}^{*} a_{i}\right)$ is the displacement operator. The (symmetric) moments are then obtained by differentiation [64],

$$
\left\langle a_{i}^{\dagger k} a_{j}^{l}\right\rangle_{s}=\left.\frac{d^{k}}{d \alpha_{i}^{k}} \frac{d^{l}}{\left(-\alpha_{j}^{*}\right)^{l}} \chi\left(\alpha_{1}, \alpha_{2}\right)\right|_{\alpha_{1}=\alpha_{2}=0},
$$

where $\langle\cdot\rangle_{s}$ is the expectation value of the symmetrized version of the operators $a_{i}^{\dagger k} a_{j}^{l}$. The evolution of the characteristic function is derived from the master equation,

$$
\frac{d}{d t} \chi\left(\alpha_{1}, \alpha_{2}\right)=\operatorname{Tr}\left\{D_{1}\left(\alpha_{1}\right) \otimes D_{2}\left(\alpha_{2}\right) \dot{\rho}\right\},
$$

together with the identities,

$$
\begin{array}{ll}
D_{i} a_{i}^{\dagger}=\left(-\frac{\alpha_{i}^{*}}{2}+\frac{d}{d \alpha_{i}}\right) D_{i}, & D_{i} a_{i}=\left(-\frac{\alpha_{i}}{2}-\frac{d}{d \alpha_{i}^{*}}\right) D_{i}, \\
a_{i}^{\dagger} D_{i}=\left(\frac{\alpha_{i}^{*}}{2}+\frac{d}{d \alpha_{i}}\right) D_{i}, & a_{i} D_{i}=\left(\frac{\alpha_{i}}{2}-\frac{d}{d \alpha_{i}^{*}}\right) D_{i} .
\end{array}
$$

with $\alpha_{i}=x_{i}+i p_{i}$ and $d / d \alpha_{i}=\left(d / d x_{i}-i d / d p_{i}\right) / 2$ using the Gaussian ansatz $\chi\left(x_{1}, p_{1}, x_{2}, p_{2}\right)=\exp \left(i \vec{P} \vec{y}-\vec{P}^{T} \bar{\sigma} \vec{P} / 2\right)$ with $\vec{P}=\left(x_{1}, p_{1}, x_{2}, p_{2}\right)^{T}$ and $\overrightarrow{\vec{y}}=\left(\bar{y}_{1}, \bar{z}_{1}, \bar{y}_{2}, \bar{z}_{2}\right)^{T}$. Since the 
Hamiltonian is purely of quadratic order, the steady-state values for the first moments always vanish $\bar{y}_{i}=0=\bar{z}_{i}$, and the system is completely described by the second moments. Writing these second moments in vector form $\vec{\sigma}=$ $\left(\bar{\sigma}_{x 1 x 1}, \bar{\sigma}_{x 1 p 1}, \bar{\sigma}_{x 1 x 2}, \bar{\sigma}_{x 1 p 2}, \bar{\sigma}_{p 1 p 1}, \bar{\sigma}_{p 1 x 2}, \bar{\sigma}_{p 1 p 2}, \bar{\sigma}_{x 2 x 2}, \bar{\sigma}_{x 2 p 2}, \bar{\sigma}_{p 2 p 2}\right)$, one may write the steady-state set of equations as $\vec{G}=\Lambda \vec{\sigma}$.
The $10 \times 10$ matrix $\underline{\Lambda}$ can be written down row-wise using $2 \bar{\Gamma}(i, j, k, l, m, n, o, p)=(-1)^{i} \Gamma$ $\left(a_{1}, a_{2}\right)+(-1)^{j} \Gamma\left(a_{1}, a_{2}^{\dagger}\right)+(-1)^{k} \Gamma\left(a_{1}^{\dagger}, a_{2}\right)+(-1)^{l} \Gamma\left(a_{1}^{\dagger}\right.$, $\left.a_{2}^{\dagger}\right)+(-1)^{m} \Gamma\left(a_{2}, a_{1}\right)+(-1)^{n} \Gamma\left(a_{2}, a_{1}^{\dagger}\right)+(-1)^{o} \Gamma\left(a_{2}^{\dagger}, a_{1}\right)+$ $(-1)^{p} \Gamma\left(a_{2}^{\dagger}, a_{1}^{\dagger}\right)$. We have

\section{ane may wite the steady-state set of equations as $G=\underline{\Lambda}$.}

$$
\begin{aligned}
& \vec{G}=\left(\Gamma\left(a_{1}, a_{1}\right)+\Gamma\left(a_{1}, a_{1}^{\dagger}\right)+\Gamma\left(a_{1}^{\dagger}, a_{1}\right)+\Gamma\left(a_{1}^{\dagger}, a_{1}^{\dagger}\right), 2 i \Gamma\left(a_{1}, a_{1}\right)-2 i \Gamma\left(a_{1}^{\dagger}, a_{1}^{\dagger}\right), \Gamma\left(a_{1}, a_{2}\right)+\Gamma\left(a_{1}, a_{2}^{\dagger}\right)+\Gamma\left(a_{1}^{\dagger}, a_{2}\right)\right. \\
& +\Gamma\left(a_{1}^{\dagger}, a_{2}^{\dagger}\right)+\Gamma\left(a_{2}, a_{1}\right)+\Gamma\left(a_{2}, a_{1}^{\dagger}\right)+\Gamma\left(a_{2}^{\dagger}, a_{1}\right)+\Gamma\left(a_{2}^{\dagger}, a_{1}^{\dagger}\right), i\left(\Gamma\left(a_{1}, a_{2}\right)-\Gamma\left(a_{1}, a_{2}^{\dagger}\right)+\Gamma\left(a_{1}^{\dagger}, a_{2}\right)-\Gamma\left(a_{1}^{\dagger}, a_{2}^{\dagger}\right)\right. \\
& \left.+\Gamma\left(a_{2}, a_{1}\right)+\Gamma\left(a_{2}, a_{1}^{\dagger}\right)-\Gamma\left(a_{2}^{\dagger}, a_{1}\right)-\Gamma\left(a_{2}^{\dagger}, a_{1}^{\dagger}\right)\right),-\Gamma\left(a_{1}, a_{1}\right)+\Gamma\left(a_{1}, a_{1}^{\dagger}\right)+\Gamma\left(a_{1}^{\dagger}, a_{1}\right)-\Gamma\left(a_{1}^{\dagger}, a_{1}^{\dagger}\right), \\
& i\left(\Gamma\left(a_{1}, a_{2}\right)+\Gamma\left(a_{1}, a_{2}^{\dagger}\right)-\Gamma\left(a_{1}^{\dagger}, a_{2}\right)-\Gamma\left(a_{1}^{\dagger}, a_{2}^{\dagger}\right)+\Gamma\left(a_{2}, a_{1}\right)-\Gamma\left(a_{2}, a_{1}^{\dagger}\right)+\Gamma\left(a_{2}^{\dagger}, a_{1}\right)-\Gamma\left(a_{2}^{\dagger}, a_{1}^{\dagger}\right)\right),-\Gamma\left(a_{1}, a_{2}\right) \\
& +\Gamma\left(a_{1}, a_{2}^{\dagger}\right)+\Gamma\left(a_{1}^{\dagger}, a_{2}\right)-\Gamma\left(a_{1}^{\dagger}, a_{2}^{\dagger}\right)-\Gamma\left(a_{2}, a_{1}\right)+\Gamma\left(a_{2}, a_{1}^{\dagger}\right)+\Gamma\left(a_{2}^{\dagger}, a_{1}\right)-\Gamma\left(a_{2}^{\dagger}, a_{1}^{\dagger}\right), \Gamma\left(a_{2}, a_{2}\right)+\Gamma\left(a_{2}, a_{2}^{\dagger}\right) \\
& \left.+\Gamma\left(a_{2}^{\dagger}, a_{2}\right)+\Gamma\left(a_{2}^{\dagger}, a_{2}^{\dagger}\right), 2 i\left(\Gamma\left(a_{2}, a_{2}\right)-\Gamma\left(a_{2}^{\dagger}, a_{2}^{\dagger}\right)\right),-\Gamma\left(a_{2}, a_{2}\right)+\Gamma\left(a_{2}, a_{2}^{\dagger}\right)+\Gamma\left(a_{2}^{\dagger}, a_{2}\right)-\Gamma\left(a_{2}^{\dagger}, a_{2}^{\dagger}\right)\right) . \\
& \underline{\Lambda}_{1}=\left(\Gamma\left(a_{1}, a_{1}^{\dagger}\right)-\Gamma\left(a_{1}^{\dagger}, a_{1}\right),-\omega_{1}, \Gamma(1,0,1,0,0,0,1,1),-i \Gamma(1,1,1,1,0,0,0,0)-(\kappa+\lambda), 0,0,0,0,0,0\right) \\
& \underline{\Lambda}_{2}=\left(\omega_{1}, 2 \Gamma\left(a_{1}, a_{1}^{\dagger}\right)-2 \Gamma\left(a_{1}^{\dagger}, a_{1}\right), i \Gamma(1,0,0,1,0,1,1,0)-(\kappa-\lambda), \Gamma(0,0,1,1,1,0,1,0),-\omega_{1},\right. \\
& \Gamma(1,0,1,0,0,0,1,1), i \Gamma(1,1,1,1,0,0,0,0)-(\kappa+\lambda), 0,0,0) \\
& \underline{\Lambda}_{3}=\left(\Gamma(0,0,1,1,1,0,1,0), i \Gamma(0,0,0,0,1,1,1,1)-(\kappa+\lambda), \Gamma\left(a_{1}, a_{1}^{\dagger}\right)-\Gamma\left(a_{1}^{\dagger}, a_{1}\right)+\Gamma\left(a_{2}, a_{2}^{\dagger}\right)-\Gamma\left(a_{2}^{\dagger}, a_{2}\right),\right. \\
& \left.-\omega_{2}, 0,-\omega_{1}, 0, \Gamma(1,0,1,0,0,0,1,1), i \Gamma(1,1,1,1,0,0,0,0)-(\kappa+\lambda), 0\right) \\
& \underline{\Lambda}_{4}=\left(-(\kappa-\lambda)+i \Gamma(0,1,1,0,1,0,0,1), \Gamma(1,0,1,0,0,0,1,1), \omega_{2}, \Gamma\left(a_{1}, a_{1}^{\dagger}\right)-\Gamma\left(a_{1}^{\dagger}, a_{1}\right)+\Gamma\left(a_{2}, a_{2}^{\dagger}\right)-\Gamma\left(a_{2}^{\dagger}, a_{2}\right), 0,0,\right. \\
& \left.-\omega_{1}, 0, \Gamma(1,0,1,0,0,0,1,1),-(\kappa+\lambda)+i \Gamma(1,1,1,1,0,0,0)\right) \\
& \underline{\Lambda}_{5}=\left(0, \omega_{1}, 0,0, \Gamma\left(a_{1}, a_{1}^{\dagger}\right)-\Gamma\left(a_{1}^{\dagger}, a_{1}\right),-(\kappa-\lambda)+i \Gamma(1,0,0,1,0,1,1,0), \Gamma(0,0,1,1,1,0,1,0), 0,0,0\right) \\
& \underline{\Lambda}_{6}=\left(0, \Gamma(0,0,1,1,1,0,1,0), \omega_{1}, 0, i \Gamma(0,0,0,0,1,1,1,1)-(\kappa+\lambda), \Gamma\left(a_{1}, a_{1}^{\dagger}\right)-\Gamma\left(a_{1}^{\dagger}, a_{1}\right)+\Gamma\left(a_{2}, a_{2}^{\dagger}\right)-\Gamma\left(a_{2}^{\dagger}, a_{2}\right),\right. \\
& \left.-\omega_{2}, i \Gamma(1,0,0,1,0,1,1,0)+(\lambda-\kappa), \Gamma(0,0,1,1,1,0,1,0), 0\right) \\
& \underline{\Lambda}_{7}=\left(0, i \Gamma(0,1,1,0,1,0,0,1)+(\lambda-\kappa), 0, \omega_{1}, \Gamma(1,0,1,0,0,0,1,1), \omega_{2}, \Gamma\left(a_{1}, a_{1}^{\dagger}\right)-\Gamma\left(a_{1}^{\dagger}, a_{1}\right)+\Gamma\left(a_{2}, a_{2}^{\dagger}\right)-\Gamma\left(a_{2}^{\dagger}, a_{2}\right),\right. \\
& 0, i \Gamma(1,0,0,1,0,1,1,0)+(\lambda-\kappa), \Gamma(0,0,1,1,1,0,1,0)) \\
& \underline{\Lambda}_{8}=\left(0,0, \Gamma(0,0,1,1,1,0,1,0), 0,0, i \Gamma(0,0,0,0,1,1,1,1)-(\kappa+\lambda), 0, \Gamma\left(a_{2}, a_{2}^{\dagger}\right)-\Gamma\left(a_{2}^{\dagger}, a_{2}\right),-\omega_{2}, 0\right) \\
& \underline{\Lambda}_{9}=(0,0, i \Gamma(0,1,1,0,1,0,0,1)+(\lambda-\kappa), \Gamma(0,0,1,1,1,0,1,0), 0, \Gamma(1,0,1,0,0,0,1,1), \\
& \left.i \Gamma(0,0,0,0,1,1,1,1)-(\kappa+\lambda), \omega_{2}, 2 \Gamma\left(a_{2}, a_{2}^{\dagger}\right)-2 \Gamma\left(a_{2}^{\dagger}, a_{2}\right),-\omega_{2}\right)
\end{aligned}
$$$$
\underline{\Lambda}_{10}=\left(0,0,0, i \Gamma(0,1,1,0,1,0,0,1)+(\lambda-\kappa), 0,0, \Gamma(1,0,1,0,0,0,1,1), 0, \omega_{2}, \Gamma\left(a_{2}, a_{2}^{\dagger}\right)-\Gamma\left(a_{2}^{\dagger}, a_{2}\right)\right)
$$

for both matrix $\Lambda$ and steady-state vector $\vec{G}$. Solving this system of equations (numerically) leads to the symplectic covariance matrix. The actual covariance matrix is obtained after symplectic transformation: $\sigma_{x i x j}=$ $\bar{\sigma}_{p i p j} / 2, \sigma_{p i p j}=\bar{\sigma}_{x i x j} / 2, \sigma_{x j p i}=-\bar{\sigma}_{x i p j} / 2$. The steady-state occupation numbers are finally calculated via $\left\langle a_{1}^{\dagger} a_{1}\right\rangle_{\mathrm{ss}}=$ $\left(\sigma_{x 1 x 1}+\sigma_{p 1 p 1}-1\right) / 2$.

\section{APPENDIX B: QUANTUM MUTUAL INFORMATION}

The quantum mutual information for a Gaussian system can be calculated from the covariance matrix as $I(\sigma)=f(a)+f(b)-f\left[n_{-}(\sigma)\right]-f\left[n_{+}(\sigma)\right][65]$ with $a=$ $\sqrt{\operatorname{det}(\alpha)}, b=\sqrt{\operatorname{det} \beta}, f(x)=(x+1 / 2) \ln (x+1 / 2)-(x-$
$1 / 2) \ln (x-1 / 2), n_{\mp}(\sigma)=\sqrt{\left[\Delta(\sigma) \mp \sqrt{\Delta(\sigma)^{2}-4 \operatorname{det} \sigma}\right] / 2}$, $\Delta(\sigma)=\operatorname{det} \alpha+\operatorname{det} \beta+2 \operatorname{det} \gamma$ for the covariance matrix defined as $\sigma_{i j}=\left\langle x_{i} x_{j}+x_{j} x_{i}\right\rangle / 2, x_{i}=\left(x_{1}, p_{1}, x_{2}, p_{2}\right)$. In this form, the submatrices of interest are $\sigma=\left[(\alpha, \gamma),\left(\gamma^{T}, \beta\right)\right]$.

\section{APPENDIX C: QUANTUM LANGEVIN EQUATIONS}

The quantum Langevin equation is derived in the Heisenberg picture [3]. This approach has the advantage that it does not involve strong approximations as is the case for quantum master equations [42-45]. On the other hand, the drawback is that it cannot easily be solved, in general, as the corresponding differential equations are operator differential equations in Hilbert space. For Gaussian systems, it can, 
however, be solved using matrix methods [3]. The steady-state solution can, thus, be obtained by matrix inversion in Fourier space [12], $M(v) \vec{a}(v)+\vec{a}_{\text {in }}(v)=0$ with the two vectors $\vec{a}(v)=\left[\tilde{a}_{1}(v), \tilde{a}_{1}^{\dagger}(-v), \tilde{a}_{2}(v), \tilde{a}_{2}^{\dagger}(-v)\right]$ and $\vec{a}_{\text {in }}(v)=$ $\left[\sqrt{2 \gamma_{1}} \tilde{a}_{1, \text { in }}(v), \sqrt{2 \gamma_{1}} \tilde{a}_{1, \text { in }}^{\dagger}(-v), \sqrt{2 \gamma_{2}} \tilde{a}_{2, \text { in }}(v), \sqrt{2 \gamma_{2}} \tilde{a}_{2, \text { in }}^{\dagger}(-v)\right]$. The matrix $M$ is explicitly given by

$M(v)$

$$
=\left(\begin{array}{cccc}
-i \nu+i \omega_{1} & 0 & i \kappa & i \lambda \\
0 & -i \nu-i \omega_{1} & -i \lambda & -i \kappa \\
i \kappa & i \lambda & -i \nu+i \omega_{2} & 0 \\
-i \lambda & -i \kappa & 0 & -i \nu-i \omega_{2}
\end{array}\right)+\bar{\gamma},
$$

with $\bar{\gamma}=\operatorname{diag}\left(\gamma_{1}, \gamma_{1}, \gamma_{2}, \gamma_{2}\right)$. Inverting $M^{-1}=m$, the second moment $\left\langle a_{1}^{\dagger} a_{1}\right\rangle$ in the algebraic space is

$$
\begin{aligned}
\left\langle a_{1}^{\dagger} a_{1}\right\rangle= & \int_{-\infty}^{\infty} \int_{-\infty}^{\infty}\left\langle a_{1}^{\dagger}(v) a_{1}\left(v^{\prime}\right)\right\rangle e^{i\left(v-v^{\prime}\right) t} / 2 \pi d v d v \\
= & (1 / \pi) \int_{-\infty}^{\infty} \gamma_{1}\left(\left|m_{11}\right|^{2} N\left(v, \beta_{1}\right)\right. \\
& \left.+\left|m_{12}\right|^{2}\left[N\left(-v, \beta_{1}\right)+1\right)\right]+\gamma_{2}\left(\left|m_{13}\right|^{2} N\left(v, \beta_{2}\right)\right. \\
& \left.+\left|m_{14}\right|^{2}\left[N\left(-v, \beta_{2}\right)+1\right)\right] d v
\end{aligned}
$$

and similar expressions for all other second moments.

The Gibbs state expectation values may in addition be evaluated by the diagonalization of the Hamiltonian given above. In general, any quadratic expectation value in the $a_{i}$ algebraic space may be calculated via $\left\langle A_{i} A_{j}\right\rangle=\sum_{k \ell} S_{i k} S_{j \ell}\left\langle C_{k} C_{\ell}\right\rangle$ with $A_{i}=\left(a_{1}, a_{2}, a_{1}^{\dagger}, a_{2}^{\dagger}\right)$ and $C_{i}=\left(c_{1}, c_{2}, c_{1}^{\dagger}, c_{2}^{\dagger}\right)$. The $\left\langle C_{i} C_{j}\right\rangle \mathrm{s}$ are then given by the uncoupled oscillators with eigenfrequencies, Eq. (3) of the main text, and corresponding temperature $T$.

\section{APPENDIX D: NONEQUILIBRIUM STEADY STATES}

The deviation of the local (and, to a lesser extent, global) quantum master equations from the quantum Langevin equation does not only depend on the magnitude of the temperature-difference $\Delta T$, but also on its sign [Figs. 6(a) and 6(b)]. We first note that the local master equation leads to larger (smaller) mean occupation numbers for weak (strong) coupling both for the position-position and the rotating-wave interactions. This increase of the mean occupation number at small coupling is caused by the frequency difference between
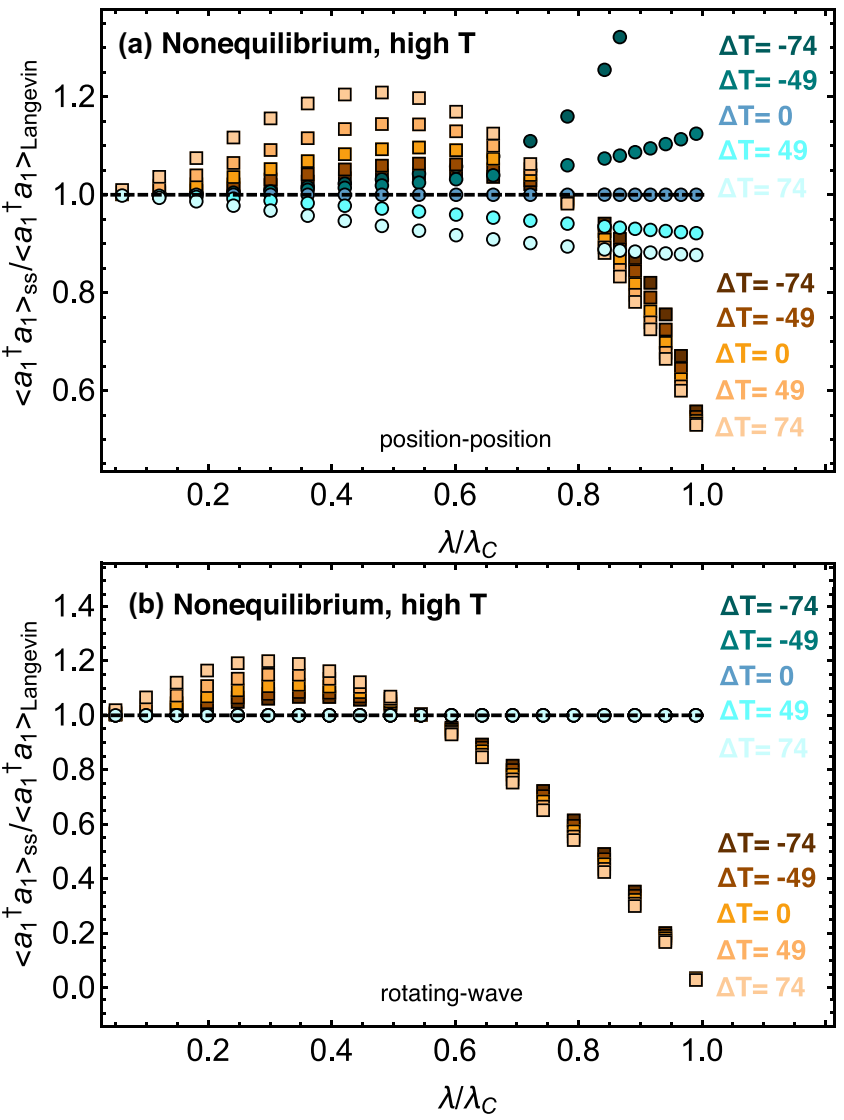

FIG. 6. Ratio of the steady-state mean occupation numbers $\left\langle a_{1}^{\dagger} a_{1}\right\rangle_{\mathrm{ss}} /\left\langle a_{1}^{\dagger} a_{1}\right\rangle_{\text {Langevin }}$ of the quantum master equation and the quantum Langevin equation as a function of $\lambda / \lambda_{c}$ for various nonequilibrium temperature-differences $\Delta T$ for (a) position-position and (b) rotating-wave interactions. Temperatures, for positive $\Delta T>$ 0 , are $T_{1}=98, T_{2}=T_{1}+\Delta T$, and for negative $\Delta T<0, T_{1}=T_{2}-$ $\Delta T, T_{2}=98$.

the oscillators $\left(\omega_{1}>\omega_{2}\right)$, which leads to a relatively larger occupation number in the second oscillator (modulated by the temperature difference), whereas its decrease is induced by strong-coupling effects.

In addition, the global master equation completely matches the Langevin equation for all $\Delta T$ for the rotatingwave interaction, whereas this is not the case for the position-position interaction: The mean occupation number is larger (smaller) than that the quantum Langevin for $\Delta T<0$ $(\Delta T>0)$.
[1] W. Pauli, Über das H-Theorem vom Anwachsen der Entropie vom Standpunkt der neuen Quantenmechanik, in Festschrift zum 60. Geburtstage A. Sommerfeld (Hirzel, Leipzig, 1928), p. 30 .

[2] H.-P. Breuer and F. Petruccione, The Theory of Open Quantum Systems (Oxford University Press, Oxford, 2002).

[3] C. Gardiner and P. Zoller, Quantum Noise (Springer, Berlin, 2004).
[4] R. Alicki and K. Lendi, Quantum Dynamical Semigroups and Applications (Springer, Berlin, 2007).

[5] A. Rivas and S. F. Huelga, Open Quantum Systems (Springer, Berlin, 2012).

[6] H. Carmichael, An Open Systems Approach to Quantum Optics (Springer, Berlin, 1993).

[7] U. Weiss, Quantum Dissipative Systems (World Scientific, Singapore, 2008). 
[8] R. Zwanzig, Nonequilibrium Statistical Mechanics (Oxford University Press, Oxford, 2001).

[9] M. A. Nielsen and I. L. Chuang, Quantum Computation and Quantum Information (Cambridge University Press, Cambridge, UK, 2000).

[10] S. Diehl, A. Micheli, A. Kantian, B. Kraus, H. P. Büchler, and P. Zoller, Quantum states and phases in driven open quantum systems with cold atoms, Nat. Phys. 4, 878 (2008).

[11] S. Diehl, A. Tomadin, A. Micheli, R. Fazio, and P. Zoller, Dynamical Phase Transitions and Instabilities in Open Atomic Many-Body Systems, Phys. Rev. Lett. 105, 015702 (2010).

[12] F. Brennecke, R. Mottl, K. Baumann, R. Landig, T. Donner, and T. Esslinger, Real-time observation of fluctuations at the driven-dissipative Dicke phase transition, Proc. Natl. Acad. Sci. USA 110, 11763 (2013).

[13] C. Carr, R. Ritter, C. G. Wade, C. S. Adams, and K. J. Weatherill, Nonequilibrium Phase Transition in a Dilute Rydberg Ensemble, Phys. Rev. Lett. 111, 113901 (2013).

[14] M. Marcuzzi, E. Levi, S. Diehl, J. P. Garrahan, and I. Lesanovsky, Universal Nonequilibrium Properties of Dissipative Rydberg Gases, Phys. Rev. Lett. 113, 210401 (2014).

[15] R. Labouvie, B. Santra, S. Heun, and H. Ott, Bistability in a Driven-Dissipative Superfluid, Phys. Rev. Lett. 116, 235302 (2016).

[16] M. Soriente, T. Donner, R. Chitra, and O. Zilberberg, Dissipation-Induced Anomalous Multicritical Phenomena, Phys. Rev. Lett. 120, 183603 (2018).

[17] T. Prosen and I. Pizorn, Quantum Phase Transition in a Farfrom-Equilibrium Steady State of an XY Spin Chain, Phys. Rev. Lett. 101, 105701 (2008)

[18] D. Karevski and T. Platini, Quantum Nonequilibrium Steady States Induced by Repeated Interactions, Phys. Rev. Lett. 102, 207207 (2009).

[19] I. Carusotto, D. Gerace, H. E. Tureci, S. De Liberato, C. Ciuti, and A. Imamoglu, Fermionized Photons in an Array of Driven Dissipative Nonlinear Cavities, Phys. Rev. Lett. 103, 033601 (2009).

[20] T. Prosen and M. Znidaric, Long-Range Order in Nonequilibrium Interacting Quantum Spin Chains, Phys. Rev. Lett. 105, 060603 (2010).

[21] T. Prosen, Open XXZ Spin Chain: Nonequilibrium Steady State and a Strict Bound on Ballistic Transport, Phys. Rev. Lett. 106, 217206 (2011).

[22] T. Prosen, Exact Nonequilibrium Steady State of a Strongly Driven Open XYZ Chain, Phys. Rev. Lett. 107, 137201 (2011).

[23] T. Prosen and E. Ilievski, Nonequilibrium Phase Transition in a Periodically Driven XY Spin Chain, Phys. Rev. Lett. 107, 060403 (2011).

[24] M. Vogl, G. Schaller, and T. Brandes, Criticality in Transport through the Quantum Ising Chain, Phys. Rev. Lett. 109, 240402 (2012).

[25] J. Cui, J. I. Cirac, and M. C. Banuls, Variational Matrix Product Operators for the Steady State of Dissipative Quantum Systems, Phys. Rev. Lett. 114, 220601 (2015).

[26] M. Foss-Feig, J. T. Young, V. V. Albert, A. V. Gorshkov, and M. F. Maghrebi, Solvable Family of Driven-Dissipative ManyBody Systems, Phys. Rev. Lett. 119, 190402 (2017).

[27] F. Carollo, E. Gillman, H. Weimer, and I. Lesanovsky, Critical Behavior of the Quantum Contact Process in One Dimension, Phys. Rev. Lett. 123, 100604 (2019).
[28] V. Popkov, T. Prosen, and L. Zadnik, Exact Nonequilibrium Steady State of Open XXZ/XYZ Spin-1/2 Chain with Dirichlet Boundary Conditions, Phys. Rev. Lett. 124, 160403 (2020).

[29] A. Levy and R. Kosloff, The local approach to quantum transport may violate the second law of thermodynamics, Europhys. Lett. 107, 20004 (2014)

[30] D. Walls, Higher order effects in the master equation for coupled systems, Z. Phys. A: Hadrons Nucl. 234, 231 (1970).

[31] H. J. Carmichael and D. F. Walls, Master equation for strongly interacting systems, J. Phys. A 6, 1552 (1973).

[32] P. D. Manrique, F. Rodriguez, L. Quiroga, and N. F. Johnson, Nonequilibrium Quantum Systems: Divergence between Global and Local Descriptions, Adv. Condens. Matter Phys. 2015, 615727 (2015)

[33] A. S. Trushechkin and I. V. Volovich, Perturbative treatment of inter-site couplings in the local description of open quantum networks, Europhys. Lett. 113, 30005 (2016).

[34] J. O. Gonzalez, L. A. Correa, G. Nocerino, J. P. Palao, D. Alonso and G. Adesso, Testing the validity of the 'local' and 'global' GKLS master equations on an exactly solvable model, Open Syst. Inf. Dyn. 24, 1740010 (2017).

[35] P. P. Hofer, M. Perarnau-Llobet, L. D. M. Miranda, G. Haack, R. Silva, J. B. Brask, and N. Brunner, Markovian master equations for quantum thermal machines: Local versus global approach, New J. Phys. 19, 123037 (2017).

[36] J. T. Stockburger and T. Motz, Thermodynamic deficiencies of some simple Lindblad operators: A diagnosis and a suggestion for a cure, Fortschr. Phys. 65, 1600067 (2017).

[37] M. T. Naseem, A. Xuereb, O. E. Mustecaplioglu, Thermodynamic consistency of the optomechanical master equation, Phys. Rev. A 98, 052123 (2018).

[38] G. De Chiara, G. Landi, A. Hewgill, B. Reid, A. Ferraro, A. J. Roncaglia and M. Antezza, Reconciliation of quantum local master equations with thermodynamics, New J. Phys. 20, 113024 (2018).

[39] M. T. Mitchison and M. B. Plenio, Non-additive dissipation in open quantum networks out of equilibrium, New J. Phys. 20, 033005 (2018).

[40] M. Cattaneo, G. L. Giorgi, S. Maniscalco, and R. Zambrini, Local versus global master equation with common and separate baths: Superiority of the global approach in partial secular approximation, New J. Phys. 21, 113045 (2019).

[41] H. Wichterich, M. J. Henrich, H.-P. Breuer, J. Gemmer, and M. Michel, Modeling heat transport through completely positive maps, Phys. Rev. E 76, 031115 (2007).

[42] A. Purkayastha, A. Dhar, and M. Kulkarni, Out-of-equilibrium open quantum systems: A comparison of approximate quantum master equation approaches with exact results, Phys. Rev. A 93, 062114 (2016).

[43] A. Rivas, A. Douglas, K. Plato, S. F. Huelga, and M. B Plenio, Markovian master equations: A critical study, New J. Phys. 12, 113032 (2010).

[44] D. Boyanovsky and D. Jasnow, Heisenberg-Langevin versus quantum master equation, Phys. Rev. A 96, 062108 (2017).

[45] M. Ludwig, K. Hammerer, and F. Marquardt, Entanglement of mechanical oscillators coupled to a nonequilibrium environment, Phys. Rev. A 82, 012333 (2010).

[46] M. Aspelmeyer, T. J. Kippenberg, and F. Marquardt, Cavity optomechanics, Rev. Mod. Phys. 86, 1391 (2014). 
[47] R. H. Dicke, Coherence in Spontaneous Radiation Processes, Phys. Rev. 93, 99 (1954).

[48] M. Tavis and F. W. Cummings, Exact Solution for an $N$ Molecule-Radiation-Field Hamiltonian, Phys. Rev. 170, 379 (1968).

[49] T. Brandes, Coherent and collective quantum optical effects in mesoscopic systems, Phys. Rep. 408, 315 (2005).

[50] P. Kirton, M. M. Roses, J. Keeling, and E. G. Dalla Torre, Introduction to the Dicke Model: From Equilibrium to Nonequilibrium, and Vice Versa, Adv. Quantum Technol. 2, 1970013 (2019).

[51] C. Emary and T. Brandes, Chaos and the quantum phase transition in the Dicke model, Phys. Rev. E 67, 066203 (2003).

[52] N. Lambert, C. Emary, and T. Brandes, Entanglement and the Phase Transition in Single-Mode Superradiance, Phys. Rev. Lett. 92, 073602 (2004).

[53] V. Sudhir, M. G. Genoni, J. Lee, and M. S. Kim, Critical behavior in ultrastrong-coupled oscillators, Phys. Rev. A 86, 012316 (2012).

[54] J.-F. Huang, J.-Q. Liao, and L.-M. Kuang, Ultrastrong JaynesCummings model, Phys. Rev. A 101, 043835 (2020).

[55] P. Forn-Diaz, L. Lamata, E. Rico, J. Kono, and E. Solano, Ultrastrong coupling regimes of light-matter interaction, Rev. Mod. Phys. 91, 025005 (2019).

[56] K. Hepp and E. Lieb, On the superradiant phase transition for molecules in a quantized radiation field: the Dicke maser model, Ann. Phys. (NY) 76, 360 (1973).
[57] Y. K. Wang and F. T. Hioe, Phase Transition in the Dicke Model of Superradiance, Phys. Rev. A 7, 831 (1973).

[58] H. Carmichael, C. Gardiner, and D. Walls, Higher order corrections to the Dicke superradiant phase transition, Phys. Lett. A 46, 47 (1973).

[59] A. Anfossi, P. Giorda, A. Montorsi, and F. Traversa, Two-Point Versus Multipartite Entanglement in Quantum Phase Transitions, Phys. Rev. Lett. 95, 056402 (2005).

[60] R. R. P. Singh, M. B. Hastings, A. B. Kallin, and R. G. Melko, Finite-Temperature Critical Behavior of Mutual Information, Phys. Rev. Lett. 106, 135701 (2011).

[61] F. C. Alcaraz and M. A. Rajabpour, Universal Behavior of the Shannon Mutual Information of Critical Quantum Chains, Phys. Rev. Lett. 111, 017201 (2013).

[62] G. De Tomasi, S. Bera, J. H. Bardarson, and F. Pollmann, Quantum Mutual Information as a Probe for Many-Body Localization, Phys. Rev. Lett. 118, 016804 (2017).

[63] C. Walsh, P. Sémon, D. Poulin, G. Sordi, A. M. S. Tremblay, Local Entanglement Entropy and Mutual Information across the Mott Transition in the Two-Dimensional Hubbard Model, Phys. Rev. Lett. 122, 067203 (2019).

[64] S. Campbell, G. De Chiara, M. Paternostro, Equilibration and nonclassicality of a double-well potential, Sci. Rep. 6, 19730 (2016).

[65] A. Serafini, F. Illuminati, S. De Siena, Symplectic invariants, entropic measures and correlations of Gaussian states, J. Phys. B: At., Mol. Opt. Phys. 37, L21 (2004). 